\title{
Colloidal hard spheres: cooking and looking †
}

\author{
Roel P. A. Dullens \\ DOI: $10.1039 / \mathrm{b} 607017 \mathrm{e}$
}

In this article we highlight our recent work on the development of crosslinked core-shell polymethyl-methacrylate colloids and their application as colloidal model hard spheres for quantitative confocal microscopy. Moreover, we demonstrate that synthesizing colloids (cooking) does not only lead to the final core-shell particles, but also to "intermediate-product-particles", which are interesting in their own right and offer additional possibilities for various physical experiments (looking). In particular, we focus on the application of crosslinked latex particles as microgel particles, non-spherically shaped particles as model particles for shape-induced geometrical frustration and the final core-shell particles for the direct measurement of thermodynamic properties using quantitative real-space confocal microscopy.

\section{Introduction}

It is safe to say that hard spheres can be considered as the "fruit flies" of statistical mechanics, where the hard sphere potential is extensively used as a reference potential. ${ }^{1}$ The reason is that at relatively high number densities, excluded volume interactions tend to dominate in all thermally excited matter: atoms, molecules, macromolecules and colloids. $^{1,2}$ The hard sphere potential consists of an infinite repulsion if the

Van't Hoff Laboratory for Physical and Colloid Chemistry, Debye Institute, Utrecht University, Padualaan 8, $3584 \mathrm{CH}$, Utrecht,

The Netherlands

$\uparrow$ Present address: 2 Physikalisches Institut, Universität Stuttgart, Pfaffenwaldring 57, 70569 Stuttgart, Germany. E-mail: r.dullens@ physik.uni-stuttgart.de spheres overlap, while the potential energy is zero otherwise. Because the hard sphere system is athermal, the number density $\rho$ is the only relevant control parameter. A commonly used measure for the number density is the volume fraction of hard spheres $\phi \equiv \rho v$, with $v$ the volume of a particle.

Whether or not hard spheres exhibit a disorder-order transition has long been the subject of a lively debate. In 1914 Bridgman argued that repulsive forces alone could produce an ordered solid. ${ }^{3}$ Later Kirkwood et al. suggested the existence of a freezing transition at sufficiently high densities. ${ }^{4,5}$ Computer simulations of Wood and Jacobson (Monte Carlo) and Alder and Wainwright (molecular dynamics) provided the first evidence for the

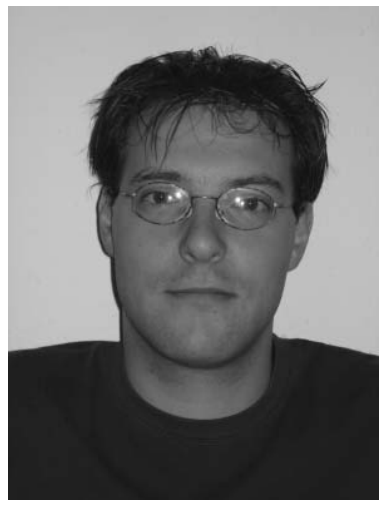

Roel Dullens
Roel Dullens obtained his MSc in chemistry in 2001 (Utrecht University, the Netherlands) and his PhD in physical and colloid chemistry in 2005 (advisors: Willem Kegel and Henk Lekkerkerker, Van't Hoff Laboratory, Utrecht University, the Netherlands). He is currently working as a Humboldt Fellow in the group of Clemens Bechinger (2. Physikalisches Institut, Universität Stuttgart, Germany). His current interests include real-space studies of colloidal systems, optical tweezers, magnetic colloids and colloidal crystals. fluid-crystal transition of hard spheres. ${ }^{6,7}$ Due to the small sizes of the simulated systems, it wasn't until 1968 that the phase boundaries were accurately determined by Hoover and Ree. ${ }^{8}$ They found that the freezing and melting transitions of hard spheres are respectively located at $\phi_{\mathrm{f}}=0.494$ and $\phi_{\mathrm{m}}=0.545$. $^{8}$

Hard spheres do not only exist in computer simulations and theories. Colloidal systems provide a wide variety of shapes and interparticle interactions including the hard sphere system. The complete hard sphere phase diagram has indeed been observed in a system of sterically stabilized polymethyl methacrylate (PMMA) colloids in an apolar refractive index matching solvent. ${ }^{9}$ In addition, the existence of a disordered state above a volume fraction of 0.58 was reported. ${ }^{9,10}$ This state has been identified as a colloidal glass. ${ }^{10}$ Other commonly used colloidal hard sphere model systems are silica spheres covered with octadecyl alcohol dispersed in cyclohexane $^{11}$ and silica spheres in refractive index matched solutions with high ionic strength. ${ }^{12,13}$

Most of the great deal of work done on colloidal systems, including hard spheres, has been performed using various kinds of scattering techniques. Although the power and usefulness of these scattering methods is beyond doubt, it cannot yield information on a local level. 
About a decade ago, confocal microscopy was successfully employed to study the structure of quenched colloidal hard spheres in real-space on a single-particle level in full 3D. ${ }^{13}$ Since then, an increasing number of real-space studies of colloidal hard spheres have been reported, see e.g. refs 14-23. Indeed, confocal microscopy and quantitative image analysis place high demands on the colloidal model system being used. First of all, particles should be labeled with a fluorescent dye in order to be visualized by confocal microscopy. Second, to accurately determine the particle positions, the fluorescent signals of neighboring particles should be separated over distances larger than the resolving power of the microscope, being of the order of the wavelength of visible light. This especially desirable at the relatively high number densities that are of interest for hard spheres.

\section{Crosslinked core-shell polymethyl-methacrylate colloids}

We met the demands that confocal microscopy places on the colloidal model system by developping crosslinked PMMA particles consisting of a fluorescent core and a non-fluorescent shell. $^{24,25}$ The particles are sterically stabilized by an outer layer of poly(hydroxy stearic acid). A fluorescently labeled crosslinked PMMA core was synthesized using dispersion polymerization, a well-known method to prepare latex particles. $^{26}$ By decoupling the nucleation and the process of crosslinking, monodisperse crosslinked PMMA particles could be prepared with sizes ranging from one-hunderd nanometres to several micrometres. ${ }^{24}$ The core was made fluorescent by incorporating different fluorescent dyes that were chemically linked to the PMMA polymers: NBD (4-methyl-aminoethyl-methacrylate-7-nitroenzo-2-oxa-1,3-diazol) and RAS (rhodamine B isothiocyanate aminostyrene). ${ }^{27}$

Subsequently, the fluorescent core was covered by a relatively thick nonfluorescent shell of crosslinked PMMA latex by two sequential seeded growth steps (see Fig. $1 \mathrm{~A}-\mathrm{C}$ ). ${ }^{27}$ The thickness of the shell can be varied from tens of nanometres to a few hundred nanometres. In particular, if the total mass of the cores $m_{\mathrm{c}}$ and the total mass of the added monomers $m_{\mathrm{a}}$ is constant, the increase of the radius $\Delta R$ is only a function of the size of the cores $R_{0}$. Using a very simple argument based on mass conservation, $\Delta R$ can be predicted. If $N$ is the total number of cores, the volume of a single particle after growth $V_{1}$ is given by

$$
\begin{aligned}
V_{1} & =\frac{1}{\rho} \frac{m_{\mathrm{c}}+m_{\mathrm{a}}}{N}=V_{0}+\frac{m_{\mathrm{c}}}{N \rho} \frac{m_{\mathrm{a}}}{m_{\mathrm{c}}} \\
& =V_{0}\left(1+\frac{m_{\mathrm{a}}}{m_{\mathrm{c}}}\right),
\end{aligned}
$$

with $\rho$ the mass density and $V_{0}=m_{\mathrm{c}} /(N \rho)$ the volume of a single core. As $\Delta R=$ $R_{1}-R_{0}$ one immediately obtains:

$$
\begin{gathered}
\Delta R=\kappa R_{0} \text { with } \\
\kappa=\left(1+m_{\mathrm{a}} / m_{\mathrm{c}}\right)^{1 / 3}-1,
\end{gathered}
$$
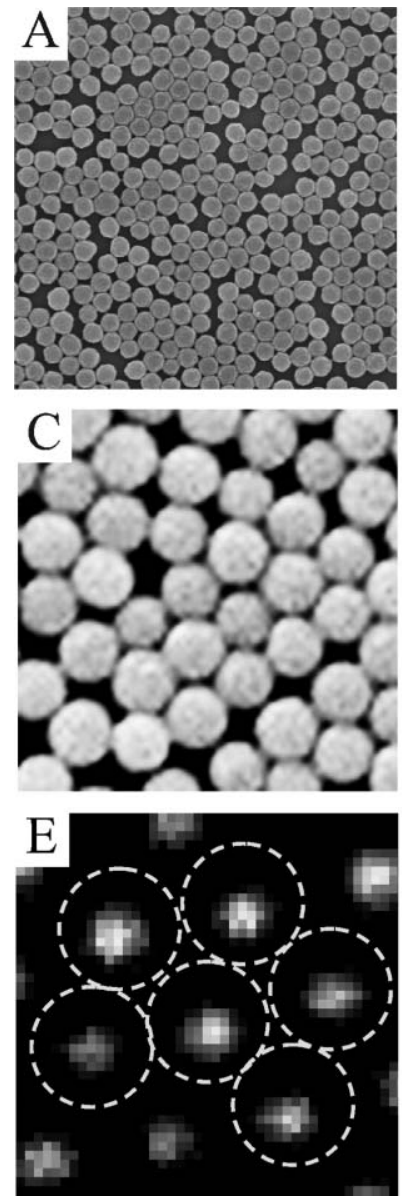

implying a linear dependence of $\Delta R$ on $R_{0}$. Applying typical values used in the growth steps, i.e. $m_{\mathrm{a}} \approx 10 \mathrm{~g}$ and $m_{\mathrm{c}} \approx 2.5 \mathrm{~g}$ (5 grams of $50 \%$ mass fraction dispersion of cores), ${ }^{24,25}$ results in $\kappa \simeq 0.71$. In Fig. 2 the increase of the radius for various growth steps with $m_{\mathrm{a}} \approx 10 \mathrm{~g}$ and $m_{\mathrm{c}} \approx 2.5 \mathrm{~g}$ is shown. The linear increase of $\Delta R$ with $R_{0}$ is evident. The slope of the linear fit is 0.73 which is remarkably close to the calculated value of 0.71 . Thus, this simple argument provides us with a fair estimate of the increase of the size and enables us to quite accurately tune the thickness of the shell. ${ }^{25}$

The crosslinking of the PMMA polymers was the crucial step in the development of the core-shell particles. Firstly, by crosslinking the polymers, a network is created preventing fluorescent
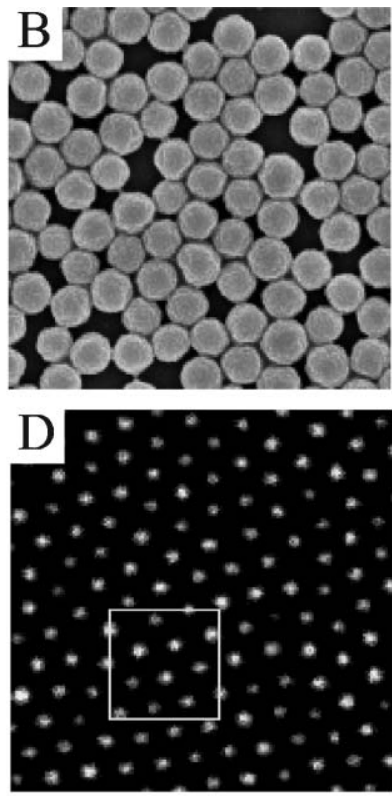

$\mathrm{F}$

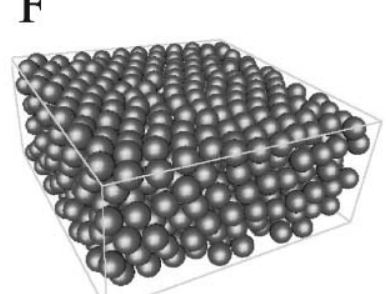

Fig. 1 Fig. 1 (A-C) Scanning electron microscopy images $\left(8 \times 8 \mu \mathrm{m}^{2}\right)$ of different stages in the preparation of RAS labeled core-shell PMMA particles: (A) core $(R=215 \mathrm{~nm})$, (B) core-shell $(R=405 \mathrm{~nm})$ and $(\mathrm{C})$ core-shell-shell $(R=635 \mathrm{~nm})$. (D) Confocal microscopy image $(16.5 \times$ $16.5 \mu \mathrm{m}^{2}$ ) of a colloidal crystal of the RAS labeled core-shell particles. In the zoom presented in (E) the complete particle diameter is indicated, showing that contacting particles are observed separately due to the core-shell structure of the particles. (F) 3D reconstruction of the colloidal crystal after all the particle positions have been located. 


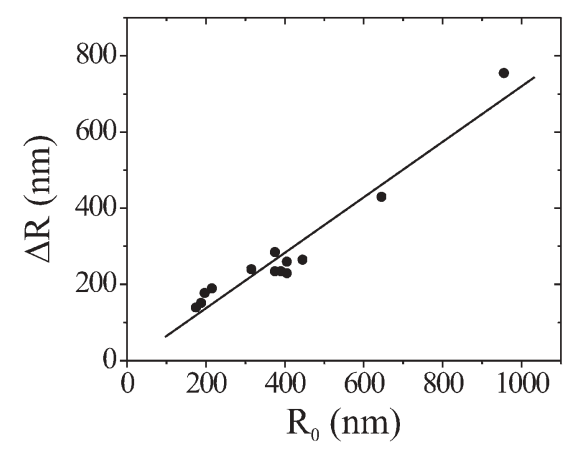

Fig. 2 The increase of the radius $\Delta R$ as a function of the core radius $R_{0}$ for different growth steps with $m_{\mathrm{a}} \approx 10 \mathrm{~g}$ and $m_{\mathrm{c}} \approx 2.5 \mathrm{~g}$ (see text). The dots represent the experimental data and the straight line is a linear fit to the data: $\Delta R=0.73 R_{0}-8.47$.

polymers from "travelling" from the core to the shell. Secondly, the network structure of the particle itself significantly suppresses the migration (if any) of the fluorescent polymers. Hence, the fluorescently labeled PMMA stays in the core, which is a prerequisite for obtaining core-shell colloids. An important property of latex-based core-shell particles is their relatively small mass density. This is particularly desirable, as it is now possible to effectively "switch off gravity" by choosing a solvent (mixture) with the same mass density as the particles. Thus by dispersing these coreshell spheres in a density and refractive index matching solvent mixture, an ideal colloidal hard sphere model system is obtained that can be used to study many fundamental problems such as freezing, melting and the glass transition using quantitative confocal microscopy. As an example we imaged a colloidal crystal in 3D and located the three dimensional coordinates of all the particles using image analysis software as described in ref. 28,29. From these positions, a computer reconstruction of the colloidal crystal is generated (Fig. 1F), illustrating the feasibility of our model system for quantitative three-dimensional confocal microscopy.

\section{Colloidal microsponges}

Contrary to non-crosslinked PMMA particles, crosslinked PMMA colloids do not fall apart in good solvents due to their spongelike network created by crosslinking the PMMA polymers. We used this property and explored the behavior of crosslinked PMMA particles in the good solvents tetrahydrofurane, chloroform and toluene using light scattering and confocal microscopy. ${ }^{24}$ The swelling of the particles was quantified by determining the swelling ratio $\alpha$ which is defined as:

$$
\alpha=\frac{R_{\text {swollen }}}{R_{\text {unswollen }}},
$$

where the particle radius in the poor solvent hexane was taken to be $R_{\text {unswollen }}$. By changing the qualilty of the solvent, the particles take up several times their own volume as is illustrated by the confocal microscopy images presented in Fig. 3A-D. While the particles are not swollen in hexane (Fig. 3A), it is obvious that the particles in toluene, chloroform and tetrahydrofurane (THF) are significantly larger (the size of the images in Fig. 3A-D is $10 \times$ $10 \mu \mathrm{m}^{2}$ ). Determining their sizes in the good solvents, shows that the swelling
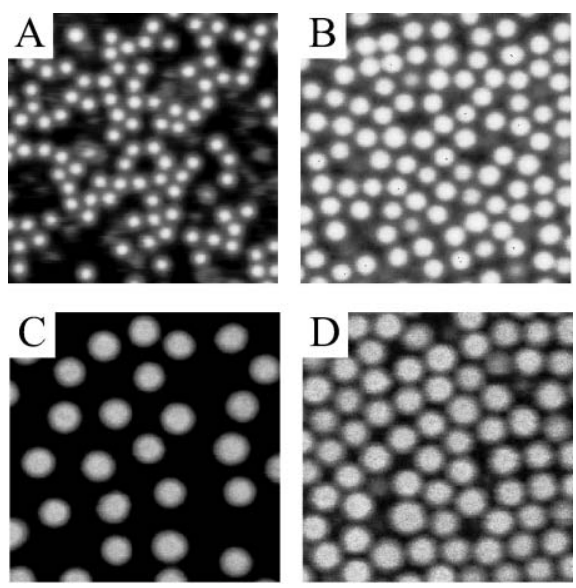

$\mathrm{E}$

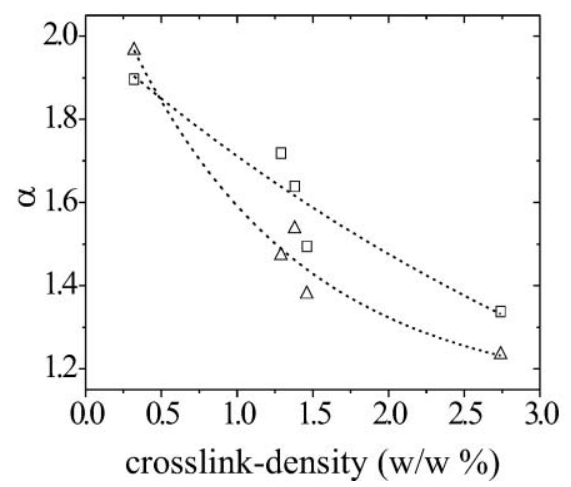

Fig. 3 Confocal images of (the same!) crosslinked PMMA particles in four different solvents: (A) hexane $(R=282 \mathrm{~nm})$, (B) toluene $(R=475 \mathrm{~nm})$, (C) chloroform $(R=535 \mathrm{~nm})$ and (D) tetrahydrofurane $(R=550 \mathrm{~nm})$. The size of the images is $10 \times 10 \mu \mathrm{m}^{2}$. (E) Swelling factor $\alpha$ as function of the crosslink-density in w/w\% in THF $(\triangle)$ and chloroform ( $\square$ ). The dashed lines are to guide the eye. 
influence of different solvents is evident from confocal microscopy studies of concentrated dispersions, which reveal clear deviations from hard sphere behavior. $^{24}$

\section{Dense packing of polyhedral colloids}

During the development of the crosslinked PMMA particles, we also observed non-spherically shaped particles, as illustrated in Fig. 4C for homogeneously fluorescent particles. $^{24}$ What precisely causes the irregular shape is not clear. It has been reported that crosslinking leads to an increased rigidity of the PMMA network, ${ }^{36}$ which might cause the irregularities in the shape of the particles. ${ }^{37,38}$ A closer characterization of the particles revealed that these "polyhedrals" are monodisperse in size, despite their non-sphericity and shapepolydispersity. ${ }^{39} \mathrm{We}$ exploited the unique properties of these particles and studied the influence of the particle shape on the structure of densely packed particles. ${ }^{39,40}$ Besides being interesting itself, it is also a rather general issue as molecular systems are monodisperse in size, but usually not spherical. Also granular systems and nanoparticles are often non-spherical.
In the experiment, we quantitatively compared single crystals of polyhedral colloids and spherical particles using confocal microscopy (see respectively Fig. 4A and B). ${ }^{39}$ Quantitative analysis of the structure of the dense packing revealed that even within single-crystallites, significant deviations from the ideal hexagonal crystal-symmetry are present. These deviations manifest themselves as bond-orientational fluctuations, which can be quantified by the distribution of angles that the nearest neighbor particles make with respect to a given reference axis. In the case of a hexagonal (single-)crystal, this 'nearest neighbor angle distribution' exhibits six peaks and the width of the peaks directly "visualizes" the bond-orientational fluctuations along each lattice direction. The difference between the distributions for the polyhedrals and spheres is clearly observed in Fig. 4D. In particular, the full-width-half-maximum (FWHM) for the polyhedrals is more than three times larger than the FWHM for the spheres. These bond-orientational fluctuations destroy the translational order in the polyhedral packing, which leads to a hexatic structure of the polyhedral colloids. A hexatic structure is characterized by long-range orientational order,

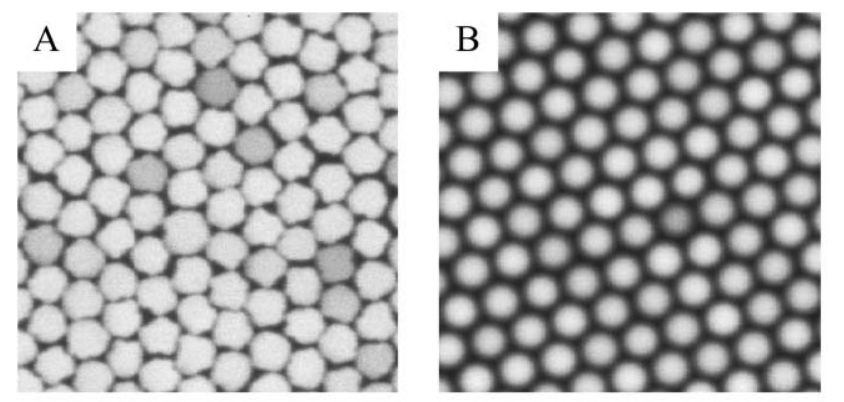

$\mathrm{C}$

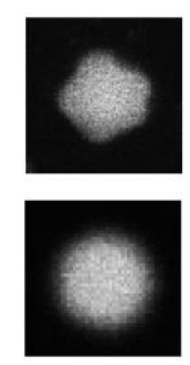

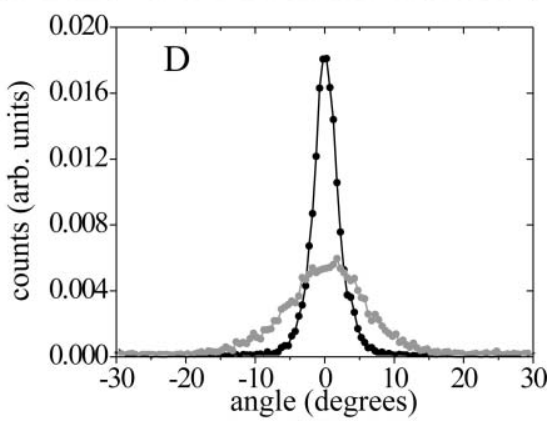

Fig. 4 Confocal microscopy images $\left(20 \times 20 \mu \mathrm{m}^{2}\right)$ showing defect-free regions of packing of polyhedrals (A) and of spheres (B). The zoomed in image (C) shows the polyhedral and spherical shape of a single particle. (D) The central peak of the nearest neighbor angle distribution (which has been shifted to $0^{\circ}$ for clarity for the polyhedrals (grey) and the spheres (black). The ratio between the FWHM widths of both peaks is 3.1. but short-range translational order, whereas a true crystal has both longrange orientational and translational order. These findings point to the large implications of only a small deviation from the spherical shape of a particle.

\section{Measuring the pressure and the chemical potential of colloidal hard spheres}

Using the specially developed crosslinked core-shell PMMA colloidal hard spheres we directly measured the pressure and the chemical potential of hard spheres. ${ }^{41}$ To this end, we used fast confocal microscopy to take three-dimensional snapshots of suspensions of the coreshell particles ranging in volume fraction from 0.05 up to 0.45 . Because of the core-shell structure of the particles, the three-dimensional positions of the particles could be accurately retrieved from the images using image analysis software as described in. ${ }^{28,29} \mathrm{~A}$ three-dimensional computer reconstruction of the system at a volume fraction of 0.25 is shown in Fig. 5A. For hard spheres, an exact theorem exists that relates certain geometrical properties of a such configuration of hard spheres to the (osmotic) pressure and the chemical potential of the system: ${ }^{42,43}$

$$
\begin{aligned}
& P=\rho k_{\mathrm{B}} T\left(1+\frac{d A_{0}}{6 V_{0}}\right), \\
& \mu=\mu^{0}-k_{\mathrm{B}} T \ln \left(\frac{V_{0}}{V}\right) .
\end{aligned}
$$

Here, $V_{0}$ and $A_{0}$ are the relevant geometrical properties and they respectively correspond to the available volume to insert an additional sphere and the surface area of that volume. In Fig. $5 \mathrm{~B}$ a 2D illustration of $V_{0}$ and $A_{0}$ is presented. Furthermore, $k_{\mathrm{B}} T$ is the thermal energy, $d$ the particle diameter, $\mu^{0}$ the ideal gas chemical potential and $V$ the total system volume. We used a lattice-method to determine $V_{0}$ and $A_{0}$ from the experimental particle configurations. ${ }^{41}$ Fig. $5 \mathrm{C}$ and D show respectively the experimentally determined pressure and chemical potential as a function of the volume fraction of spheres (circles) compared to the Carnahan-Starling equation of state (full curves). ${ }^{44}$ The observed agreement demonstrates that both the pressure and chemical potential can be measured from 

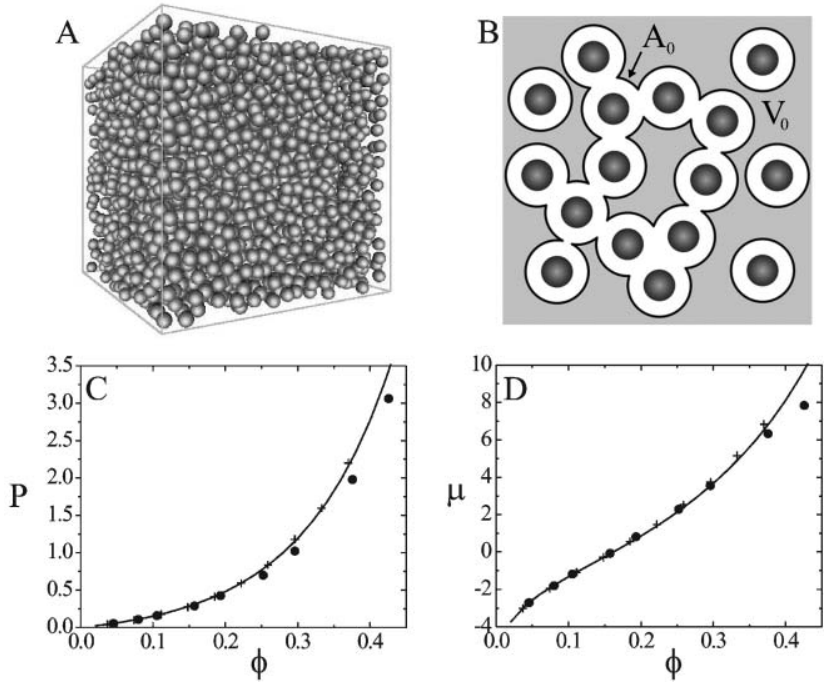

Fig. 5 (A) Three dimensional computer reconstruction of the colloidal system at $\phi=0.25$. (B) 2D illustration of $V_{0}$ (grey area) and $A_{0}$ (black lines). (C) Pressure $P$ in units of $k_{\mathrm{B}} T / v$ and (D) chemical potential $\mu$ in $k T_{\mathrm{B}}$ as a function of $\phi$. Circles denote measurements; full curves follow from the Carnahan-Starling equation of state. ${ }^{45}$ The agreement between Monte-Carlo simulations (crosses) and the full curve validates the procedure followed.

pictures alone. At higher volume fractions the deviations slightly increase, since $V_{0}$ becomes exceedingly small and many more snapshots are required to assure proper averaging. Although the main results may be unsurprisingexperiments, computer simulation and theory all agree well-this noninterfering 'visual' determination of thermodynamic quantities provides a new experimental tool to gain further insight into thermodynamic properties of fluids.

\section{Conclusions}

We have shown that cooking and looking is a fruitful combination in colloid science. In particular, we developed crosslinked PMMA colloidal model particles, which can be used for various applications. Crosslinking the PMMA facilitated the application of these particles as colloidal microsponges, which swell significantly in good solvents but do not fall apart. Furthermore, we obtained non-spherical polyhedral colloids which are used to show that only a small perturbation of the spherical shape has large implication on the structure of a colloidal crystal. Finally, by preparing colloids consisting of a fluorescent core and a non-fluorescent shell, an ideal colloidal hard sphere model system for quantitative real-space studies using fluorescence confocal microscopy is obtained. As an example we showed the direct measurement of thermodynamic properties by confocal microscopy using these coreshell particles.

\section{Acknowledgements}

Willem Kegel, Dirk Aarts, Maria Claesson, Maurice Mourad, Didi Derks, Jacob Hoogenboom, Alfons van Blaaderen and Henk Lekkerkerker are thanked for many valuable discussions and support. This work is part of the research programme of the Stichting voor Fundamenteel Onderzoek der Materie (FOM), financially supported by the Nederlandse Organisatie voor Wetenschappelijk Onderzoek (NWO).

\section{References}

1 J. D. Weeks, D. Chandler and H. C. Andersen, J. Chem. Phys., 1971, 54, 5237.

2 S. Sastry, T. M. Truskett, P. G. Debenedetti, S. Torquato and F. H. Stillinger, Mol. Phys., 1998, 95, 289.

3 P. W. Bridgman, Phys. Rev., 1914, 3, 153.

4 J. G. Kirkwood, J. Chem. Phys., 1939, 7, 919.

5 J. G. Kirkwood, E. K. Maun and B. J. Alder, J. Chem. Phys., 1950, 18, 1040.

6 W. W. Wood and J. D. Jacobsen, J. Chem. Phys., 1957, 27, 1207.

7 B. J. Alder and T. E. Wainwright, J. Chem. Phys., 1957, 27, 1208.

8 W. G. Hoover and F. H. Ree, J. Chem. Phys., 1968, 49, 3609.
9 P. N. Pusey and W. van Megen, Nature, 1986, 320, 340.

10 P. N. Pusey and W. van Megen, Phys. Rev. Lett., 1987, 59, 2083.

11 C. G. de Kruif, J. W. Jansen and A. Vrij, in Physics of Complex and Supermolecular Fluids, ed. S. A. Safran and N. A. Clark, Wiley, New York, 1987.

12 A. van Blaaderen and A. Vrij, Langmuir, 1992, 8, 2921.

13 A. van Blaaderen and P. Wiltzius, Science, 1995, 270, 1177.

14 A. van Blaaderen and P. Wiltzius, Nature, 1997, 385, 321

15 W. K. Kegel and A. van Blaaderen, Science, 2000, 287, 290.

16 E. R. Weeks, J. C. Crocker, A. C. Levitt, A. Schofield and D. A. Weitz, Science, 2000, 287, 627.

17 U. Gasser, E. R. Weeks, A. Schofield, P. N. Pusey and D. A. Weitz, Science, 2001, 292, 258.

18 J. P. Hoogenboom, D. Derks, P. Vergeer and A. van Blaaderen, J. Chem. Phys., 2002, 117, 11320 .

19 R. P. A. Dullens and W. K. Kegel, Phys. Rev. Lett., 2004, 92, 195702.

20 P. Schall, I. Cohen, D. A. Weitz and F. Spaepen, Science, 2004, 305, 1944.

21 R. P. A. Dullens and W. K. Kegel, Phys. Rev. E: Stat. Phys., Plasmas, Fluids, Relat. Interdiscip. Top., 2005, 71, 011405.

22 P. Schall, I. Cohen, D. A. Weitz and F. Spaepen, Nature, 2006, 440, 319.

23 N. B. Simeonova, R. P. A. Dullens, D. G. A. L. Aarts, V. W. A. Villeneuve, H. N. W. Lekkerkerker and W. K. Kegel, Phys. Rev. E: Stat. Phys., Plasmas, Fluids, Relat. Interdiscip. Top., 2006, 73, 041401.

24 R. P. A. Dullens, E. M. Claesson and W. K. Kegel, Langmuir, 2004, $20,658$.

25 R. P. A. Dullens, E. M. Claesson, D. Derks, A. van Blaaderen and W. K. Kegel, Langmuir, 2003, 19, 5963.

26 K. E. J. Barret, Dispersion Polymerization in organic media, Wiley, London, 1975.

27 G. Bosma, C. Pathmamanoharan, E. H. A. de Hoog, W. K. Kegel, A. van Blaaderen and H. N. W. Lekkerkerker, J. Colloid Interface Sci., 2002, 245, 292.

28 J. C. Crocker and D. G. Grier, J. Colloid Interface Sci., 1996, 179, 298.

29 J. P. Hoogenboom, P. Vergeer and A. van Blaaderen, J. Chem. Phys., 2003, 119, 3371.

30 B. E. Rodriguez and M. S. Wolfe, Macromolecules, 1994, 27, 6642.

31 E. Bartsch, S. Kirsch, T. Schere and S. Stolken, Ber. Bunsen-Ges. Phys. Chem., 1998, 102, 1597.

32 W. Schärtl, Adv. Mater., 2000, 12, 1899.

33 V. E. Shashoua and R. G. Beaman, J. Polym. Sci., 1958, 33, 101.

34 A. Kose and S. Hachisu, J. Colloid Interface Sci., 1974, 46, 460.

35 M. Wolfe and C. Scopazzi, J. Colloid Interface Sci., 1989, 133, 265.

36 R. J. R. Cairns, R. H. Ottewill, D. W. J. Osmond and I. Wagstaff, J. Colloid Interface Sci., 1976, 54, 45.

37 C. M. Tseng, Y. Y. Lu, M. S. El-Aasser and J. W. Vanderhoff, J. Polym. Sci., Part A: Polym. Chem., 1986, 24, 2995. 
38 K. Li and D. Stover, J. Polym. Sci., Part A. Polym. Chem., 1993, 31, 2473.

39 R. P. A. Dullens, M. C. D. Mourad, D. G. A. L. Aarts, J. P. Hoogenboom and W. K. Kegel, Phys. Rev. Lett., 2006, 96, 028304 .
40 R. P. A. Dullens, V. W. A. Villeneuve, M. C. D. Mourad and W. K. Kegel, (submitted).

41 R. P. A. Dullens, D. G. A. L. Aarts and W. K. Kegel, Proc. Natl. Ac. Sci. U. S. A., 2006, 103, 529.
42 B. Widom, J. Chem. Phys., 1963, 39, 2808.

43 R. J. Speedy, J. Chem. Soc. Faraday Trans. 2, 1980, 76, 693

44 N. F. Carnahan and K. E. Starling, J. Chem. Phys., 1969, 51, 635.

\section{Find a SOLUTION}

\section{... with books from the RSC}

\section{Choose from exciting textbooks, research level books or reference books in a wide range of subject areas, including:}

- Biological science

- Food and nutrition

- Materials and nanoscience

- Analytical and environmental sciences

- Organic, inorganic and physical chemistry

\section{Look out for 3 new series coming soon ...}

- RSC Nanoscience \& Nanotechnology Series

- Issues in Toxicology

- RSC Biomolecular Sciences Series

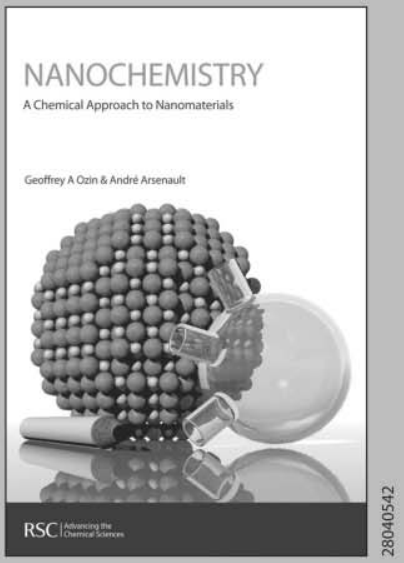

Www.rsc.org/books 